\title{
Filtering Noisy 802.11 Time-of-Flight Ranging Measurements
}

\author{
Andreas Marcaletti \\ ETH Zurich \\ Zurich, Switzerland
}

\author{
Maurizio Rea \\ ETH Zurich \\ Zurich, Switzerland
}

\author{
Domenico Giustiniano \\ IMDEA Networks Institute \\ Madrid, Spain
}

\author{
Vincent Lenders \\ Armasuisse \\ Thun, Switzerland
}

\author{
Aymen Fakhreddine \\ IMDEA Networks Institute \& \\ UC3M \\ Madrid, Spain
}

\begin{abstract}
Time-of-Flight (ToF) echo techniques have been proposed as a way to estimate the range between regular Wi-Fi stations. Recent works either did not address practical questions for deployability, or made evaluations in basic setups, or used advanced 802.11 hardware designs. We build an approach solely deployed using ToF measurements and relying on software access point (AP) upgrades of simple commercial off-the-shelf 802.11 chipsets. Our solution filters noisy measurements collected by WiFi chipsets of six dollars each, it has been tested across different and heterogeneous setups and testbeds, and has the potential to enable ToF ranging in every Wi-Fi chipsets.
\end{abstract}

\section{Categories and Subject Descriptors}

C.2.1 [Computer-Communication Networks]: Network Architecture and Design-Wireless Communication

\section{Keywords}

Indoor Localization; 802.11; Time of Flight; Design; Implementation; Evaluation

\section{INTRODUCTION}

In the attempt to find an alternative to error-prone signalstrength based ranging measurements $[3,5,10,13,16]$, Timeof-Flight (ToF) echo techniques have recently received attention by the research community. The underlying principle of ToF is that the distance between two devices is estimated using the time that the signal travels between two devices, with the advantage of being much less susceptible to the diversity of the obstacles between the devices $[8,14]$. The intuition is that electromagnetic waves travel at a speed that is close to the speed of light for most propagation media in typical indoor environments, and thus the signal propagation speed is fairly independent on the environment, obstacles, etc.

\footnotetext{
Permission to make digital or hard copies of all or part of this work for personal or classroom use is granted without fee provided that copies are not made or distributed for profit or commercial advantage and that copies bear this notice and the full citation on the first page. Copyrights for components of this work owned by others than ACM must be honored. Abstracting with credit is permitted. To copy otherwise, or republish, to post on servers or to redistribute to lists, requires prior specific permission and/or a fee. Request permissions from permissions@acm.org.

CoNEXT'14, December 2-5, 2014, Sydney, Australia.

Copyright 2014 ACM 978-1-4503-3279-8/14/12 ...\$15.00.

http://dx.doi.org/10.1145/2674005.2674998.
}

Despite of these advantages, ToF measurements are very sensitive to noise since, at the speed of light, a measurement error of $1 \mu \mathrm{s}$ already results in a distance estimation error of 300 meters. This noise is further exacerbated when using Commercial Off-The-Shelf (COTS) WiFi devices for a costeffective and wide-spread implementation. Recent efforts could increase the accuracy of ToF at the cost of leveraging advanced hardware designs available in more recent $\mathrm{WiFi}$ chipsets [14]. However, most of the Access Points (APs) in the world are still deployed with older hardware, and it is economically not possible to change all the APs with newer hardware in the near future (and also not appealing in terms of electronic garbage).

We leverage the existing 802.11 protocol timing specification (and nothing more than that) to build a system that runs on COTS APs to estimate the distance to target stations. We rely on a customized firmware operating in the core of the 802.11 MAC state machine of a low-cost WiFi chipset (cost per unit of less than six dollars). To deal with the intrinsic challenges of noisy ToF measurements, we develop an adaptive filter which manages to predict the distance despite the large noise introduced by the devices and the multipath reflections in indoor environments.

Our approach has been tested across various setups and testbeds. We show that our filtering technique needs just a few samples to estimate the distance range with a median error of $1.7-2.4 \mathrm{~m}$ and a 80 -percentile error of $3.7-5.8 \mathrm{~m}$, comparable to [14] (median error of $\approx 1 \mathrm{~m}$ and 80-percentile error of $\approx 5 \mathrm{~m}$ ) that required advanced inputs from the hardware such as channel state information per antenna. Our solution further outperforms recent works such as [8].

Given its simplicity, the principles discussed in our work can be applied to any old and new 802.11 AP already deployed, just with software upgrades.

\section{BACKGROUND AND CHALLENGES}

This section describes the basics of $\mathrm{WiFi}$ ToF ranging and highlights various real-world challenges that arise when applying this technique with simple off-the-shelf devices.

\subsection{WiFi ToF Ranging Technique}

While traditional ToF-based echo techniques as employed in radar systems rely on uncoded RF signals and their reflections, WiFi echo techniques use regular frames of communication. In WiFi communication, every DATA frame is acknowledged by the receiver with an ACK frame. Since 


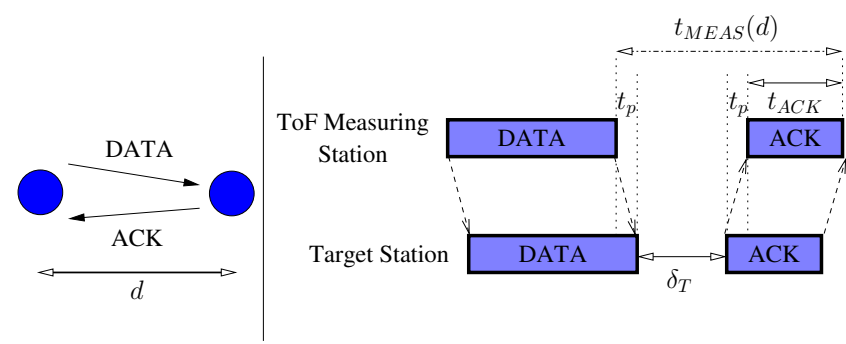

Figure 1: Principle of the Wi-Fi ToF echo technique. In the example, $\delta_{T}$ offset is originated at the target device, and it depends on the hardware delay to schedule the ACK at the target device. Not shown in the figure, time offset may be also generated at the measuring device.

the interframe time between the DATA and ACK frames is fixed by the 802.11 standard (the Short InterFrame Symbol, SIFS, time), the delay between DATA and ACK frames can be used to infer the distance between two nodes. However, ToF-based ranging estimation is affected by severe noise and has thus long been considered impracticable for WLAN localization. Back then, [12] suggested that the accuracy of spacing between a DATA and ACK frame that are defined to be separated by a SIFS time is up to $2 \mu \mathrm{s}$, equal to $600 \mathrm{~m}$ of error, and too high for indoor localization. However, the technique was studied in $[6,9,11,15]$ (subjected to variable jitter and very high dispersion), and later refined in $[7,8,14]$ showing that better accuracy is achievable than the wortcase bound dictated by the maximum SIFS tolerance.

ToF ranging can be formulated as follows. If $d$ is the distance between a local station and a target, the measured time-of-flight $t_{M E A S}(d)$ between a sent DATA frame and a received ACK frame is expressed as

$$
t_{M E A S}(d)=2 \cdot t_{P}(d)+t_{A C K}+\delta,
$$

where $t_{P}(d)$ is the signal propagation time between the transmitter of the DATA frame and the target (channel reciprocity is assumed), $t_{A C K}$ is the time needed to transmit the ACK, $\delta$ is an offset depending on the target and local sender. The distance to the target device is then inferred as

$$
\hat{d}_{n}=\frac{c}{2} \cdot\left(t_{M E A S}(d)-t_{A C K}-\delta\right),
$$

where $c$ is the speed of signal propagation which is close to the speed of light in air. Assuming that $N$ consecutive samples $\left\{\hat{d}_{n}\right\}$ are collected, the distance between the local station and the mobile device is finally estimated as:

$$
\hat{d}=f\left(\hat{d}_{n}\right),
$$

where $f$ is either the expected value on the input data or any other estimator.

Firmware integration. To alleviate any unnecessary source of noise or instability, the time measurements $t_{M E A S}(d)$ have to work as close as possible to the radio hardware. The best location to fully control the measurement is therefore in the firmware of the $\mathrm{WiFi}$ radio chipset, rather than in the driver as proposed in [8]. To measure $t_{M E A S}(d)$ in the firmware, we have customized the open-source 802.11 openFWWF firmware ${ }^{1}$. This firmware is written in assembler

\footnotetext{
${ }^{1}$ http: //www . ing. unibs.it/openfwwf/
}

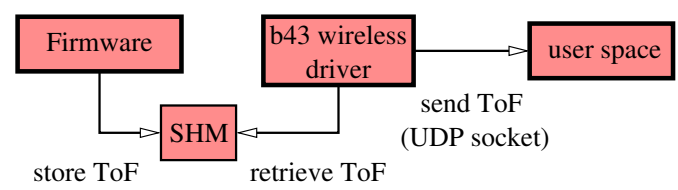

Figure 2: ToF measurement architecture. The figure shows the interaction between firmware, driver and user space. Measured data from the firmware is transferred to the driver through shared memory. Buffered ToF measurements are then sent to user space.

and runs on off-the-shelf 802.11 Broadcom chipsets, such as the ones widely used in Linksys APs. Our customized firmware reports $t_{M E A S}(d)$ for each successful transmission of an 802.11 DATA frame. The timing is regulated by the general purpose timer, running on the wireless card's internal clock at a rate of $88 \mathrm{MHz}$. The timer starts to count clock cycles just after the 802.11 processor sets up a register to indicate that a frame has been sent. Once the ACK frame has been received (or the ACK timeout has elapsed), another register is updated and the timer gets stopped. Every time a measurement is made, the firmware writes $t_{M E A S}(d)$ into a defined address of the shared memory (SHM). The measurement architecture is shown in Fig. 2. Since the driver has also access to the shared memory block, it can retrieve the measurement every time an ACK is received ${ }^{2}$. In the driver, we gather additional data about the incoming ACK such as the data rate, MAC addresses, etc, and store them all in a buffer. Once this buffer is full or a timeout elapsed, the data is transferred to the user space with the help of UDP sockets.

\subsection{Real-world Challenges}

In the real-world, ToF measurements are affected by large noise coming from the timing imprecision of off-the-shelf WiFi devices. The offset $\delta$ is given by:

$$
\delta=\delta_{T}+\delta_{L}
$$

where $\delta_{T}$ and $\delta_{L}$ are the offsets of target station and local sender, respectively. In addition, the multipath signal propagation characteristics of complex indoor environments play an important role to $\mathrm{ToF}$ measurements. In the following, we describe the main sources of noise and how they affect the accuracy of ToF ranging.

Target and measuring noise. The 802.11 standard specifies the SIFS time between the reception of a DATA and the transmission of an ACK at the receiver as a fixed interval. In $802.11 \mathrm{~b}$, for example, this time is specified as $10 \mu \mathrm{s}$ [2]. However a relatively high tolerance of $1 \mu \mathrm{s}$ is tolerated which can result in significant noise and distance estimation errors up to 300 meters if the target would fully exploit this specified tolerance level. While most chipsets may not fully exploit this tolerance, the dispersion is still quite significant. To illustrate this, Fig. 3 on the left represents the resulting dispersion of a typical Broadcom WiFi chipset as target device. The shown histogram was obtained by estimating the distance $\hat{d}_{n}$ according to Eq. (2) for 10000 packets. To

\footnotetext{
${ }^{2}$ We operate in promiscuous mode which allows us to know in the driver when an ACK has been received and thus a new data is available in the SHM.
} 


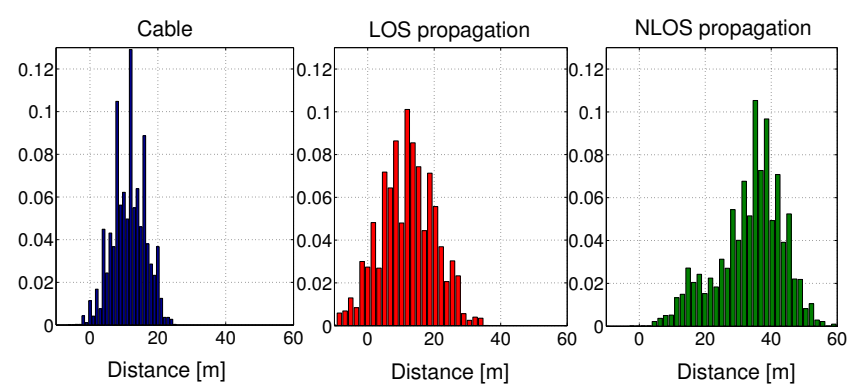

Figure 3: Noise introduced by ToF ranging. Tests with controlled environment (Cable) show that the there are sources of large dispersion in the estimation. Tests over the air (LOS and NLOS propagation) show that the distribution greatly depends on the channel conditions. All the tests are in addition subject to quantization noise and other spurious noise sources.

avoid any dispersion from environmental effects, the measurements were performed over a coaxial cable of 13.5 meters length. As we can see, there is heavy noise in the measurement setup that leads to distance estimations ranging from 0 to 25 meters.

Environmental noise. It is well known that signal propagation in complex indoor environments is subject to multipath effects in which multiple copies of the transmitted signal arrive at the receiver over different reflected paths. It is even possible that the direct component is entirely attenuated and the signal is received only over indirect paths. Since signals that travel over indirect paths will take longer to arrive at the receiver, they introduce an error in the distance estimation when considering the time-of-flight. This situation is shown in Fig. 3 in the middle and on the right where the same experiment as on the left was repeated but for a line-of-sight (LOS) and non-line-of-sight (NLOS) signal propagation link over omnidirectional antennas. The dispersion spans a range of 40 and 60 meters for the LOS and NLOS links respectively. In addition, the NLOS link shows a skewed distribution, suggesting that the signals are received from different propagation paths over the duration of the experiment. Multipath effects must therefore be taken into consideration in order not to overestimate the distance when dealing with reflected signal propagation paths. Finally, multipath may also happen in LOS links, and thus a method robust to the propagation conditions must be designed.

Additional sources of noise. Off-the-shelf WiFi chipsets have not been designed to provide accurate ToF measurements. Additional noise therefore comes from the coarse clock resolution of the radios. For example, the Broadcom chipset operates with a reference clock of $88 \mathrm{MHz}$, corresponding to a maximal distance resolution of 1.7 meters. In addition to this quantization noise, off-the-shelf chipsets introduce all sorts of considerable additional noise. As we could see in the histograms of Fig. 3, the shape of the distribution is far from being smooth despite using 10000 samples to create the histograms, suggesting that the radios must have some bias when measuring the time. The measurement

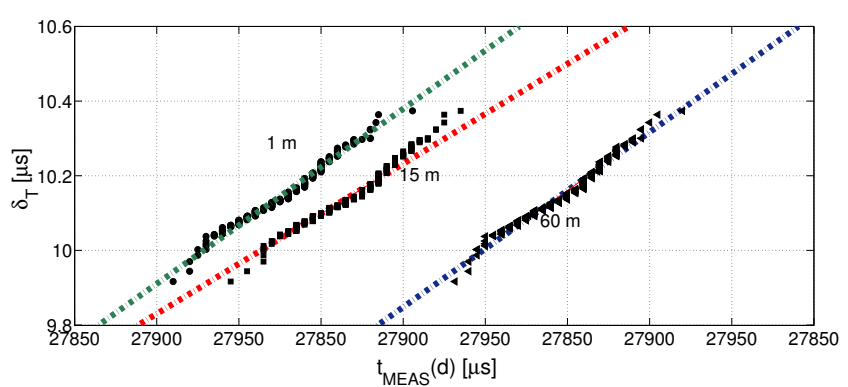

Figure 4: QQ plots for LOS links. The plots show that the error of the local offset has a negligible impact on the noise of $t_{M E A S}(d)$.

noise must therefore also be factored in to estimate the distance.

\section{TARGET AND MEASURING NOISE}

To dissect the relative importance of the noise of the target station with respect to the local noise, we use a wideband oscilloscope (Infiniium 90000A Oscilloscope) with a fast sampling rate of $10 \mathrm{GS} / \mathrm{s}$ to measure the target offset $\delta_{T}$. A horn antenna is connected to the oscilloscope which serves as a passband filter for the $2.4 \mathrm{GHz}$ band. The target device is in close proximity to the horn antenna, so that radio signals of received 802.11 DATA and transmitted 802.11 ACK are immediately captured by the oscilloscope, and effects of signal reflections are minimized. The noise of our highend oscilloscope can be regarded as low such that reported measurements are not affected by any measuring noise but dominated by the target noise [4].

We use above setup to statistically compare the effective ToF to the measured time $t_{M E A S}(d)$ as being locally reported by our firmware on the Broadcom chipset. To compare both distributions, we use the method of the quantilequantile (QQ) plot. We collect samples in LOS settings at $d=\{1,15,60\} \mathrm{m}$. Results are summarized in Fig. 4. We observe a linear pattern, which indicates that the noise of the target station and the data measured locally by the 802.11 firmware have very similar distributions. Hence, the dispersion of the local offset has a negligible impact on the noise of $t_{M E A S}(d)$, and most of the dispersion comes from the target device. This further demonstrates that our approach to implement the ToF measurement in the firmware allows to minimize the impact of the local dispersion of the noise for LOS links with limited multipath. In the next section, we look at the impact of richer multipath.

\section{ENVIRONMENTAL NOISE}

Environmental noise is tricky to deal with. The reasons are the following:

- multipath reflections introduce a skewness of the sample distribution which cannot be easily smoothed out by averaging over a set of biased samples,

- the target noise introduces large offset noise which adds to the noise created by multipath,

- environmental noise is highly unpredictable as the reflected paths are location-specific. 
We therefore need an adaptive filter that is calibrated to the actual multipath characteristics of the experienced location-specific environment.

Our goal is to design a filter that takes a series of $N$ consecutive measurements $\left\{\hat{d}_{1}, \hat{d}_{2}, \ldots, \hat{d}_{N}\right\}$, and selects the p-percentile $\hat{d}(p)$ of the series that minimizes the estimation error. Intuitively, in absence of multipath, the median $p=50$ could fairly represents the distance, while taking the median in presence of multipath reflections will cause an over-estimation of the distance due to the added traveled distance of the signals. By selecting a percentile $p<50$ to estimate the distance, we can therefore counterbalance those biased values in the estimation process.

\subsection{Data Integrity}

First, to guarantee data integrity in our evaluation, we removed sequences of samples which experienced undesired side effects for a systematic evaluation. In particular, for statistical relevance of the results, consecutive ToF measurements are supposed to be independent and identically distributed (i.i.d.). While this assumption holds in most tests, this condition does not hold in general. We perform cable tests and they show that the autocorrelation is very low for lag greater than zero. We observe however that there are links with high autocorrelation when DATA is transmitted over the air. This could be explained with the presence of more than one path between transmitter and receiver, which may imply that the circuitry continuously jumps between two states in an attempt to tune the frame synchronization. We therefore discard those sequences which were subject to a high absolute lag-one autocorrelation (above 0.2 ) for the analysis. Removing measurements with a high autocorrelation is a valid assumption in the real-world as this kind of test can easily be performed by the AP.

\subsection{Estimating Multipath Noise}

The second step is to estimate the noise caused by multipath reflections on a link. Since we cannot directly measure the individual multipath components at the signal-level on off-the-shelf WiFi radios, we propose to use a higher-level estimator $f$ for this. We explored various options in an extensive evaluation in one of our testbeds (Testbed I, Fig. 7) with all links with low lag-one autocorrelation $(83 \%$ of the links). As estimators, we considered the first three moments (median, standard deviation, and skewness) for the ToF as well as for the Received Signal Strength Indicator (RSSI). For each of these six estimators, we evaluate two variants, leading to a total of twelve candidate estimators. In the first variant, we determine the moments directly on the raw samples. In the second variant, we attempt to pre-filter obvious outliers that arise from the device-related measurement noise prior determining the moments. These outliers are filtered out applying the Thompson Tau technique, a statistical method for deciding whether to keep or discard samples based on the expected value and the expected deviation of the sequence of samples.

We evaluate the precision of these estimators by determining their correlation to the optimal percentile $p_{o p t}$, defined as the percentile that provides the minimum absolute distance estimation error as:

$$
p_{o p t}=\underset{0 \leq p \leq 0.5}{\operatorname{argmin}}|d-\hat{d}(p)|
$$

\begin{tabular}{r|cc}
\hline & unfiltered & pre-filtered \\
\hline median of RSSI & 0.62 & 0.63 \\
standard deviation of RSSI & 0.04 & 0.05 \\
skewness of RSSI & 0.23 & 0.24 \\
median of ToF & 0.76 & 0.76 \\
standard deviation of ToF & 0.19 & 0.21 \\
skewness of ToF & 0.20 & 0.51 \\
\hline
\end{tabular}

Table 1: Absolute value of Pearson correlation coefficient between different moments of the RSSI and ToF versus the optimal percentile $p_{o p t}(0=$ no correlation, $1=$ maximal correlation).

that is, the a-posteriori optimal percentile for each link given the true distance of each link in our experiments.

To quantify the correlation between the different moments and $p_{\text {opt }}$, we use the Pearson correlation coefficient on the entire set of links of Testbed I. The Pearson correlation coefficient is an indicator of the linear correlation of the variables, where absolute values close to zero indicate a low correlation and absolute values close to one represent a high linear dependence of two variables. A value close to one thus indicates that a moment is a good estimator to predict a percentile that will filter out the multipath noise effectively.

Table 1 shows the resulting correlation coefficient for all twelve variants. The best correlation is provided by the median of the ToF, followed by the median of the RSSI and the skewness of the ToF. All other moments have a correlation coefficient below 0.5 which indicates a low correlation. Most of the moments profit from pre-filtering to remove the outliers. In particular, the skewness of the ToF increases from 0.20 to 0.51 and is therefore considerably better when pre-filtering the outliers.

One may wonder why the skewness of the ToF has a worse correlation than the median ToF. Intuitively, the skewness of the distribution should be a good indicator of the multipath, given that links with strong reflected (delayed) components are left-skewed, with $p_{\text {opt }}$ smaller than for right-skewed link. Our results suggest that the combined device-related noise of the receiver and the measuring station have a strong negative effect on the correlation on the skewness. This is reflected in the pre-filtered version of the skewness which has a considerably better correlation than the unfiltered version. In contrast, the median of the ToF is much more robust to this device-related noise and therefore outperforms the skewness. In addition, it reflects the tendency of having more links affected by multipath for longer distances.

\subsection{Filter Design}

Motivated by the good correlation of the median ToF and the optimal percentile, we designed a filter that relies on this correlation to select an appropriate percentile of the sample ToF measurements when estimating the distance. The filter relies on a linear model which is derived from the empirical distribution of the median ToF versus $p_{o p t}$. We call it offline calibration, since it would be usually performed before actual tests. This distribution on all the 207 links of Testbed I is shown in the top of Fig. 5. We note that the value of the optimal percentile is widely distributed between $0 \leq p_{o p t} \leq$ $50 \%$. Therefore, it does not exist one value of percentile 

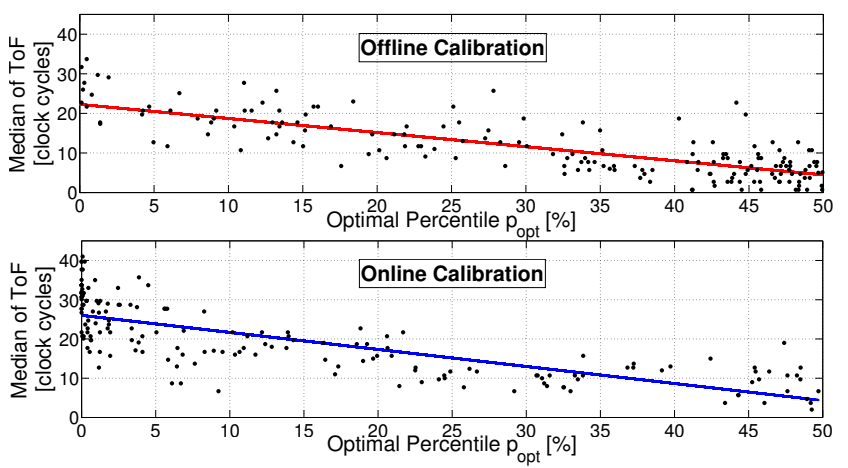

Figure 5: Median ToF versus optimal percentile $p_{o p t}$ for all links of Testbed I - Offline and online calibration.

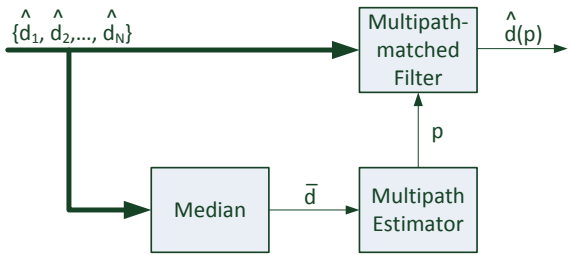

Figure 6: Adaptive filter design.

$p$ that it is optimal, but it rather changes from link to link. We therefore perform a linear regression on these data points and obtain the linear link-dependent model, as represented by the continuous line in the figure. Since the correlation coefficient of the median ToF versus the optimal percentile is not improved by pre-filtering outliers, we apply this model on the raw, unfiltered samples. (For the estimator based on the skewness of ToF we instead use the pre-filtered version).

Since it is desirable to avoid offline calibration, we then run the same methodology measuring the median ToF between pairs of APs, which can be performed online (as long as the distance between APs is known a priori). The results are shown in the bottom of Fig. 5. We find that the calibration results in a very similar linear regression.

We illustrate our filter design in Fig. 6. In practice, our filter works as follows. For a given series of $N$ consecutive measurements $\left\{\hat{d}_{1}, \hat{d}_{2}, \ldots, \hat{d}_{N}\right\}$, we first determine the median ToF, $\bar{d}$. The median ToF is then used to estimate the amount of multipath using a linear model. The output of the estimator is the percentile value $p$. We apply linear interpolation to the sequence of measurements $\left\{\hat{d}_{1}, \hat{d}_{2}, \ldots, \hat{d}_{N}\right\}$ and select the $p$-percentile $\hat{d}(p)$ of the series that minimizes the error.

\section{EVALUATION METHODOLOGY}

This section presents the system and environmental setup we used to evaluate the performance of our filter.

\subsection{System Setup}

AP infrastructure. We have build a prototype ToF system to evaluate its performance in real-world conditions. Our system is based on COTS APs from Soekris (net5501

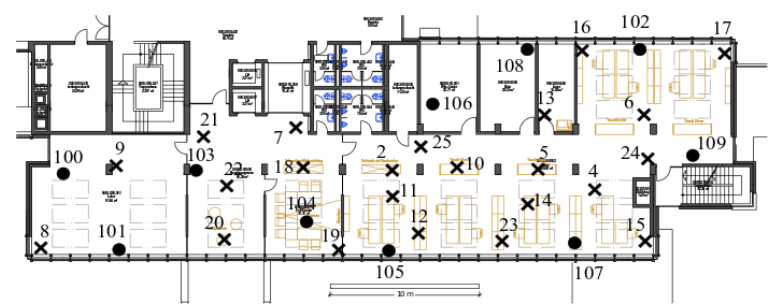

Figure 7: Testbed I. Circles correspond to AP locations and crosses to target locations.

embedded machine with a $500 \mathrm{MHz}$ AMD Geode LX single chip processor). The APs are equipped with Broadcom AirForce54G 4318 mini PCI type III cards which we operate with our customized firmware and b43 driver presented in Section 2.1. As operating system on the APs we use Ubuntu server 10.04 with a Linux kernel version 2.6.32.60. In order to operate the embedded device as WLAN AP, we use the software suite hostapd. The APs are connected to the central localization unit over Gigabit Ethernet.

Target devices. As target devices we use Dell Inspiron 5150 laptops equipped with Broadcom AirForce54G 4318 mini PCI type III cards. The operating system on the laptops is Ubuntu 10.04 with its original Linux kernel version 2.6.32-21.32. The wireless cards are operated in client mode.

\subsection{Deployment Scenarios}

Testbed deployment. We perform experiments in three indoor testbeds deployed in different environments. We deploy 10 APs in Testbed I and III and 9 APs in Testbed II. Testbed I and Testbed II are office environments. The environment of Testbed $\mathrm{I}$ is shown in Fig. 7 and it covers a surface of almost $1000 \mathrm{~m}^{2}$. We use 25 randomly selected locations (marked with a cross) to test our algorithms. We conducted tests over two different days, with some position repeated again with different location of some furniture, and collected a total of 207 wireless links. Testbed II features 180 links and it covers a smaller space of around $200 \mathrm{~m}^{2}$. The target station is in 20 different positions. Testbed III has been deployed at the facilities of the IEEE/ACM IPSN Microsoft Indoor Localization Competition [1]. The testbed features 200 links and it covers $320 \mathrm{~m}^{2}$. The target device is placed at 20 different positions across two rooms and a hallway.

In all the testbeds there exists a mixture of line-of-sight and non-line-of-sight wireless links. The testbeds also contain several propagation obstructions, including concrete walls, tables and glasses. All experiments are conducted with other active WLAN networks. We operate the three testbeds on a fixed frequency channel of the $2.4 \mathrm{GHz}$ ISM band. The PHY automatic selection rate is active, such that the measurements include probes sent at different rates.

\section{EVALUATION RESULTS}

This section evaluates the performance of different ranging estimators in our testbeds.

\subsection{Filter Performance}

Distance Ranging Accuracy. To test our filter, we have evaluated its performance on all the links of Testbed I. For each link, we first compute the distance estimation error with 20 samples, and then calculate the average error 


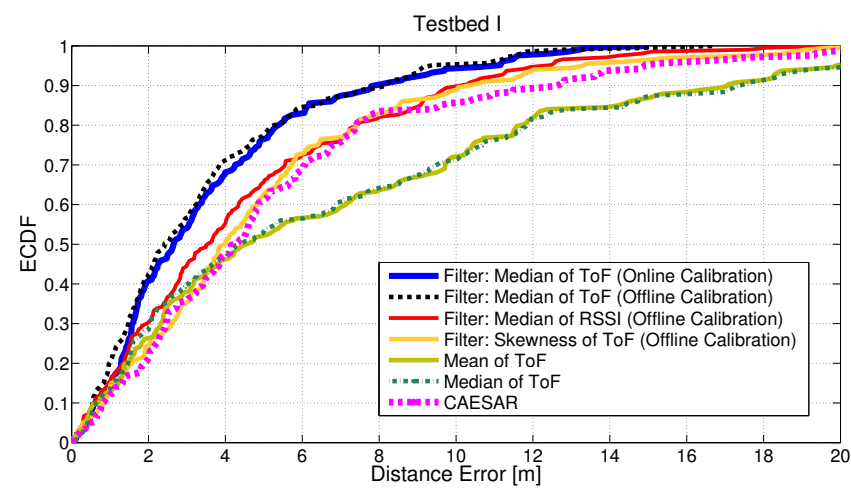

Figure 8: ECDF of the distance estimation error for our adaptive filter compared to other schemes (Testbed I). For each scheme, we use sequences of 20 samples.

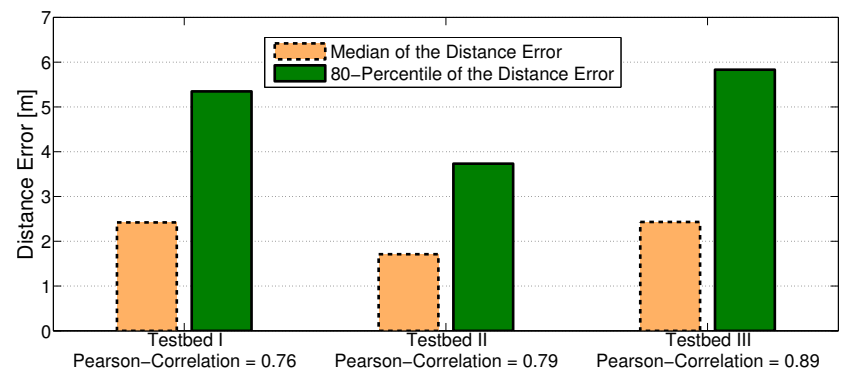

Figure 9: Performance in the three different testbeds.

using 500 sequences. We consider our filter that uses the median ToF to estimate the optimal percentile, and two other versions of the filter that rely on the median of the RSSI (pre-filtered) and the skewness of the ToF (pre-filtered), as they provided the second- and third-best correlation coefficients in Section 4.2. In addition, we also provide the error for naive approaches such as the mean and median of the ToF (without any filter).

Figure 8 shows the Empirical Cumulative Distribution Function (ECDF) of the distance ranging error. Our three new estimators clearly outperform the mean or the median. The mean and median have roughly equal estimation error. Their median error is approximately $4.5 \mathrm{~m}$ and the 80 -percentile error is approximately $11.8 \mathrm{~m}$. The error of the filter that uses the median RSSI slightly outperforms the skewness of the ToF. This is not surprising since Table 1 shows a higher correlation coefficients of the filter with median RSSI. The best performance is achieved with our filter using the median ToF. In Testbed I, we obtain a median error of $2.4 \mathrm{~m}$ and a 80-percentile error of $5.3 \mathrm{~m}$.

Comparison with [8]. Fig. 8 also shows that our three estimators outperform CAESAR, introduced in [8], which achieves a median error of $4.3 \mathrm{~m}$ and a 80 -percentile error of $7.4 \mathrm{~m}$. Our evaluation of CAESAR is also very consistent to the one recently presented in the indoor evaluation of [14].

Online calibration. We then compare online versus offline environmental calibration for the filter of median of

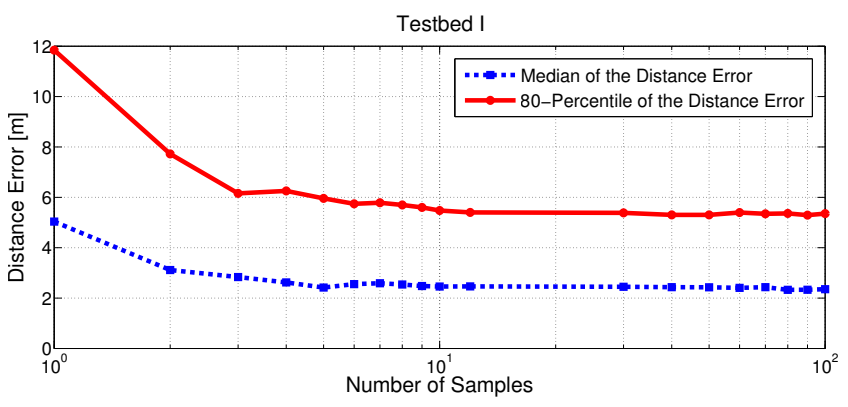

Figure 10: Median and 80-percentile error of our estimator that filters the noise based on the median of ToF. Results are provided for different number of samples.

ToF (similar results are achieved with our other filters). As reported in Fig. 8, the online calibration achieves similar results with respect to the offline tests, with median error of $2.6 \mathrm{~m}$ and a 80 -percentile error of $5.4 \mathrm{~m}$. Thus, the environmental calibration can be executed online without significant performance loss.

Robustness to different environments/testbeds.

Fig. 9 shows the median and 80-percentile of the distance error for the three different testbeds using sequences of 20 samples. As shown in the x-label of the figure, we measure a high Pearson correlation coefficient between the median of ToF and the optimal percentile $(0.76-0.89)$. The median distance error is in the range $1.7-2.4 \mathrm{~m}$ and the 80 -percentile error is in the range $3.7-5.8 \mathrm{~m}$. Concluding, the estimator is robust across different environments.

Impact of Number of ToF Samples. A further parameter we evaluate with regard to our filter is the number of ToF measurement samples $N$ that are used for the estimation of the distance. Figure 10 shows the error for our filter that relies on the median of the ToF as a function of the number of samples. It is remarkable that the error is stable with five or more samples for the median of the distance error, and with ten or more samples for the 80 -percentile of the distance error.

\section{CONCLUSION}

We have designed a cost-effective ranging technique such that it does not require any special hardware, special antennas, or software-defined radio architectures. Our solution can be deployed as a software upgrade to current WLAN infrastructures. While our best performing estimator may be a bit counter-intuitive, we have shown its robust performance in a variety of different setups and testbeds. Since ToF-based WLAN localization is still at its infancy, we believe that further research in this area might help to even further improve the accuracy of our results in the future.

\section{Acknowledgments}

We are grateful to Francesco Gringoli for sharing the openFWWF code and supporting the project with his enlightening comments. The work was supported by Armasuisse. The research leading to these results has received funding from the European Research Council under the EU's Seventh Framework Programme, ERC Grant Agreement no. 617721. 


\section{REFERENCES}

[1] Microsoft indoor localization competition, http://research.microsoft.com/en-us/events/ ipsn2014indoorlocalizatinocompetition/.

[2] IEEE std 802.11-2012 (revision of IEEE std 802.11-2007), IEEE standard for information technology, 2012.

[3] Bahl, P., and Padmanabhan, V. RADAR: an in-building RF-based user location and tracking system. In Proc. IEEE INFOCOM'00 (Mar. 2000).

[4] Bourchas, T., Bednarek, M., Giustiniano, D., AND Lenders, V. Poster abstract: Practical limits of wifi time-of-flight echo techniques. In Proceedings of the 13th International Symposium on Information Processing in Sensor Networks (Piscataway, NJ, USA, 2014), IPSN '14, IEEE Press, pp. 273-274.

[5] Chintalapudi, K., Padmanabha Iyer, A., And Padmanabhan, V. N. Indoor localization without the pain. In In Proc. of ACM MobiCom '10 (2010), pp. 173-184.

[6] Ciurana, M., Barcelo-Arroyo, F., And IZQUIERDO, F. A ranging system with IEEE 802.11 data frames. In Radio and Wireless Symposium, 2007 IEEE (2007), pp. 133-136.

[7] Gallo, P., Garlisi, D., Giuliano, F., Gringoli, F., AND Tinnirello, I. WMPS: A positioning system for localizing legacy 802.11 devices. In Transactions on Smart Processing and Computing, (October 2012).

[8] Giustiniano, D., And Mangold, S. CAESAR: carrier sense-based ranging in off-the-shelf 802.11 wireless lan. CoNEXT '11, ACM, p. 10.

[9] Golden, S. A., And Bateman, S. S. Sensor measurements for Wi-Fi location with emphasis on time-of-arrival ranging. IEEE Trans. Mobile Comput. 6, 10 (Oct. 2007), 1185-1198.

[10] Goswami, A., Ortiz, L. E., And Das, S. R. Wigem: A learning-based approach for indoor localization. CoNEXT '11, ACM, pp. 3:1-3:12.

[11] Günther, A., And Hoene, C. Measuring round trip times to determine the distance between wlan nodes. NETWORKING'05, Springer-Verlag, pp. 768-779.

[12] Li, X., Pahlavan, K., Latva-aho, M., And YliantTila, M. Comparison of indoor geolocation methods in dsss and ofdm wireless lan systems. In IEEE Fall VTC 2000 (2000), vol. 6, pp. 3015-3020 vol.6.

[13] Lim, H., Kung, L.-C., Hou, J., And Luo, H. Zero-configuration, robust indoor localization: Theory and experimentation. In INFOCOM 2006. 25th IEEE International Conference on Computer Communications. Proceedings (April 2006), pp. 1-12.

[14] Mariakakis, A. T., Sen, S., Lee, J., and Kim, K.-H. Sail: Single access point-based indoor localization. In Proceedings of the 12th Annual International Conference on Mobile Systems, Applications, and Services (New York, NY, USA, 2014), MobiSys '14, ACM, pp. 315-328.

[15] McCrady, D., Doyle, L., Forstrom, H., Dempsey, T., and Martorana, M. Mobile ranging using low-accuracy clocks. Microwave Theory and Techniques, IEEE Transactions on 48, 6 (2000), 951-958.

[16] Youssef, M., And Agrawala, A. The horus wlan location determination system. MobiSys '05, ACM, pp. 205-218. 